\title{
Knowledge, Attitude and Practices of Breastfeeding-An Extensive Review
}

\author{
Sultana $J^{1}$, Hossain $M R^{2}$, Begum $N N F^{3}$, Nazme $N l^{4}$
}

\begin{abstract}
Breastfeeding has been accepted as the most vital intervention for reducing infant mortality and ensuring optimal growth and development of children. Breastfeeding is also considered as the most economical and easily accessible complete nutrition for every new born child ${ }^{1}$. Poor breastfeeding practices are widespread. It is estimated that sub-optimal breastfeeding, especially non-exclusive breastfeeding in the first 6 months of life, results in 1.4 million deaths and $10 \%$ of the disease burden in children younger than 5 years of age ${ }^{2}$.
\end{abstract}

Reviews of studies from developing countries showed that infants who were not breast fed were 6 to 10 times more likely to die in the first months of life than infants who are breast fed ${ }^{3}$. The World Health Organization has stated that in 2000 , only $16 \%$ of mothers in Pakistan exclusively breast feed for a period of three months, as compared to other developing countries where the ratio is higher like Bangladesh (46\%), India (37\%), and Sri Lanka $(84 \%)^{4}$. More than $15 \%$ of 24 lakh child deaths could be averted in India by optimal breastfeeding practices ${ }^{5}$. The key to successful breastfeeding is Information, Education and Communication (IEC) strategies aimed at behavior change ${ }^{6}$.

The World Health Organization (WHO) and United Nations Children's Fund (UNICEF) strongly recommends exclusive breastfeeding (EBF) for the first six months of life ${ }^{7}$. Variables that may influence breastfeeding include race, maternal age, maternal employment, level of education of parents, socio-economic status, insufficient milk supply, infant health problems, method of delivery, maternal interest and other related related factors ${ }^{8,9}$. Over the last decade, overwhelming scientific evidence supporting the integral role of breastfeeding in the survival, growth and development of a child, as well as the health and well-being of a mother has come to light ${ }^{10}$. Different studies were designed at national and international level to explore the knowledge, attitude and practices (kap) towards breastfeeding among postnatal mothers and factors that determine them ${ }^{1}$.

\section{Knowledge, attitude and practice}

In different studies, knowledge of breastfeeding were assessed among mothers by asking multiple questions regarding onset and duration of breastfeeding, advantage and disadvantage of breast milk or colostrum, problems regarding breastfeeding etc. Plans to attend classes related to breastfeeding during pregnancy, driving force for starting breastfeeding and self-image of the participants were the main questions addressing attitude in different studies. Practices were assessed in almost all the studies by observing the number of participants who breastfed exclusively or stopped breastfeeding early or delivered formula feed in spite of breast milk etc.

\section{Exclusive breastfeeding}

According to the World Health Organization (WHO), "Exclusive breastfeeding means that the infant receives only breast milk. No other liquids or solids are given - not even water - with the exception of oral rehydration solution, or drops/syrups of vitamins, minerals or medicines ${ }^{11}$." To enable mothers to establish and sustain exclusive breastfeeding for six months, the WHO and the United Nations Children's Emergency Fund (UNICEF) recommend:

1. Col Jesmin Sultana, MBBS, DCH, MCPS, FCPS, Classified Child Specialist and Neonatologist, CMH, Dhaka 2. Maj Gen Muhammad Rabiul Hossain, MBBS, MCPS, FCPS, FRCP, Director General Medical Services, Bangladesh Armed Forces, Dhaka 3. Brig Gen Nurun Nahar Fatema Begum, MBBS, FCPS, FRCP, FACC, FSCAI, Head of the Department of Paediatrics, CMH and AFMC, Dhaka 4. Maj Nure Ishrat Nazme, MBBS, FCPS, Classified Child Speciallist, CMH, Dhaka. 
- "Initiation of breastfeeding within the first hour of life;

- Exclusive breastfeeding - that is, the infant only receives breast milk without any additional food or drink, not even water;

- Breastfeeding on demand - that is, as often as the child wants, day and night;

- No use of bottles, teats or pacifiers ${ }^{12}$."

\section{Advantage of breastfeeding for children}

The WHO recommends that for the first six months of life, infants should be exclusively breastfed to achieve optimal growth, development and health. Thereafter, infants should receive nutritionally adequate and safe complementary foods, while continuing to breast feed for up to two years or more ${ }^{13,14}$. Optimal breastfeeding of infants under one year could prevent around a million deaths of children under-five in the developing world ${ }^{15}$.

Breast milk is cheap, clean, accessible, safe and contains antibodies that help protect infants and boost immunity. Consequently, breastfeeding contributes to protect infants from common childhood illnesses such as diarrhea and pneumonia, the two primary causes of child mortality worldwide ${ }^{10}$. Breastfeeding is also associated with a reduced risk of otitis media, sudden infant death syndrome, necrotizing enterocolitis, obesity, and hypertension ${ }^{16}$. Breastfeeding lowers the risk of allergic disease and protects against chronic diseases like bronchial asthma, inflammatory bowel disease,childhood cancer etc ${ }^{17}$. Beyond the immediate benefits for children, breastfeeding contributes to a lifetime of good health. It has been demonstrated that adults who were breast fed as infants have better blood pressure and cholesterol profiles, in addition to lower weights and incidence of type-2 diabetes. There is evidence that people who were breastfed perform better on intelligence tests ${ }^{17,18}$.

\section{Advantage of breastfeeding for mother}

For the mother, breastfeeding is economical; breast milk is always available, clean and at the right temperature. Breastfeeding also delays the return of fertility and reduces the risk of developing breast and ovarian cancers ${ }^{17}$. Breastfeeding has also been shown to develop mother-infant bonding amongst other benefits. Lack of breastfeeding increases risk of ovarian and breast cancer, osteoporosis and UTI to mother $^{19}$.

\section{BMS Code: International Code of BMS}

The International Code of Marketing of Breast-milk Substitutes was adopted in May 1981 by the Health assembly of WHO and UNICEF. It sets standards for health care systems, health care workers, and formula distributors regarding the promotion of formula in comparison to breast feeding ${ }^{20}$.

\section{The International Code of Marketing of BMS}

Code prohibits violations in terms of:

- Advertisement of BMS

- Commercial promotion in health facility

- Company contact with mothers

- Donation of product to health facility include teats and bottles

- Free samples

- Gift to mothers

- Gift to health workers

- Inadequate labeling

- Promotion in shops and sponsorship

\section{BMS Law Bangladesh 2013}

Name of the act- The Breast-milk Substitutes, Infant Foods, Commercially Manufactured Complementary Foods and the Accessories there of (Regulation of Marketing) Act, 2013. Enacted in- 22nd September, 2013. Bangladesh enacted its first ever law regarding breast milk substitutes (BMS) to protect and promote the breast feeding of children almost 30 years ago. The ordinance was named "Breast Milk Substitutes (Regulation of Marketing) Ordinance, 1984." In 2013 Bangladesh Government has come up with a stricter act abolishing the previous ordinance ${ }^{21}$.

\section{Status of breast feeding-International perspective} Rate of breast feeding: Multiple studies were conducted in different parts of the world to assess knowledge, practice and attitude of breast feeding among different types of people. Breastfeeding rates vary in different countries ${ }^{22}$. Globally the prevalence of exclusive breastfeeding in developing countries ${ }^{23}$ increased from 33\% in 1995 to $39 \%$ in 2010.

Initiation and duration of breast feeding: The knowledge regarding time of initiation of breastfeeding, colostrum feeding, duration of exclusive breastfeeding, expressed breast milk and continuation of breastfeeding in sick baby is very poor in India ${ }^{1}$. Poverty and ignorance are the main reasons for poor breast feeding practice, which is one of the major causes of 
malnutrition among infants ${ }^{24}$. In another study. Jordanian women had a positive attitude regarding breastfeeding but work place and short maternity leaves had a negative impact on breastfeeding ${ }^{25}$. A large number of mothers are not practicing exclusive breastfeeding in rural Jamaica. The impact of knowledge about and attitude toward breastfeeding on the duration of exclusive breastfeeding is also poorly understood ${ }^{26}$.

Trends in breast feeding differ among African countries. For example, while only $37 \%$ of all infants in Mozambique younger than six months are exclusively breastfed, breastfeeding has been found to be universal in Zambia, and almost every child is breastfed after birth ${ }^{27,28}$. In many African societies, exclusive breastfeeding is influenced by various socio-economic, cultural and biological factors. In Malawi and Mozambique, in addition to breastfeeding, most women start practising mixed feeding, including the introduction of water, traditional medicines and porridge to their babies diets, before they reach six months of age $\mathrm{e}^{27,28}$. Another study in Nigeria, dicovered that no baby was exclusively breastfed and prelacteal feeds ranging from water, formula or herbal tea were given by all the mothers ${ }^{29}$.

While assessing the level of knowledge, attitude and practices (KAP) of breastfeeding among working mother, a study in Malaysia revealed that knowledge and positive attitude alone is insufficient in promoting exclusive breastfeeding. Short duration of maternityleave (2 months in Malaysia) restrain mothers from exclusive breast feeding. However, most of them still believed that plain water could prevent dehydration and breastfeeding is not a popular method of family planning $^{30}$.

The breastfeeding initiation rate within the first 48 hours, was found to be $100 \%$ among female high school teachers and health care workers of Saudi Arabia $^{16,18}$. The data in various studies of India ${ }^{31}$ shows that initiation rates vary from 16 to $54.5 \%$. But sustained exclusive breastfeeding up to six months was present in only $8.3 \%$ of participants reported by Al-Binali ${ }^{16}$ which was higher than US studies and lower than a Ugandan study (49.8\%) and another Saudi study $(15.9 \%)^{18,32}$. The most common reason for early stopping of breastfeeding (within 1 year) was insufficiency of breast milk described in a Saudi study which is similar to other studies $^{16,18,33}$. Breast milk insufficiency, adverse work related issues and a high percentage of babies receiving readymade liquid formula while still in hospital were the main reasons for the very low rate of exclusive breastfeeding among female school teachers in Saudi Arabia reported by Al-Binali ${ }^{18}$.

Knowledge of health care workers: The knowledge and the attitudes of medical and paramedical personnel working in urban and rural health centers can greatly affect breastfeeding practice. A Nigerian study illustrates substantial and unacceptable knowledge gaps among frontline cadres of health workers $^{17}$. Similar knowledge gap has been identified by Sadoh et al in their study of medical women's breastfeeding practices ${ }^{34}$. In one study, women in Malawi seemed less knowledgeable about proper breastfeeding practices largely as a result of poor quality of counselling from hospital staff ${ }^{35}$. A cross sectional survey of medical students from first year to final year at a private medical college in Pakistan showed inadequate knowledge of the students regarding initiation and duration of breastfeeding ${ }^{4}$.

Despite the numerous advantages of breastfeeding, very few health workers in Nigeria are aware of important benefits like encouraging bonding between mother and child, the benefit as a means of family planning, rather some implicates breastfeeding as a cause of diarrheal disease. This is a critical finding given that this erroneous belief could lead to dangerous misinformation and counseling of breastfeeding mothers ${ }^{17}$. In an Indian study, it was seen that the knowledge about benefits and technique of expressed breastmilk was very low. Taking into account the increasing proportion of working mothers, this is a very essential element, which needs to be taught to expectant and postnatal mothers by health care workers ${ }^{1}$.

Relation with maternal socio-demography: Higher breastfeeding scores correlated with higher maternal age in an Indian study ${ }^{1}$. Similar finding has been reported in many other studies ${ }^{36,37}$. In Jordan lesseducated women were more likely to breastfeed than women with higher education levels. In contrast, a positive association was found between breastfeeding 
and maternal education status in Western communities and developed countries $^{38}$. In a Nigerian study, giving colostrum to babies was seen more amongst mothers with higher levels of education but exclusive breastfeeding were not influenced by the mother's level of education ${ }^{17}$. In an Indian study, higher socio-economic status and maternal occupation with professionals correlated with better breastfeeding scores. But this could be because of the educational status of the mothers from higher socio economic class ${ }^{1}$.

Delivery of colostrum: The unique nutritional and antibody properties of colostrum and the disadvantages to those infants not fed with colostrum are now well recognized and documented. But many mothers do not know about the advantage of delivering colostrum to the newborn ${ }^{39}$. Some medical students are even not aware of its benefit rather they think colostrum to be harmful for the baby ${ }^{4}$. There is also practice of discarding colostrum and replacing it with a wide range of pre-lacteal feeds and late initiation of breastfeeding $^{17}$. This finding was contrary to what was reported by $\mathrm{Al}$-Jassir et al. in 2006, where very few mothers claimed ignorance of the benefits of colostrum $^{40}$.

\section{Status of breast feeding-Bangladesh perspective}

Breastfeeding is the fundamental component of the child-survival strategy ${ }^{41}$. It has been estimated that $13 \%$ under five children deaths could be reduced each year if babies were exclusively breastfed for the first six months followed by appropriate complementary feeding at least in the first year of life $^{42}$. In Bangladesh, studies have focused on protective effect of exclusive breastfeeding against most causal agents of diarrhoea mostly due to cholera and shigellosis ${ }^{43,44}$.

Although Bangladesh is traditionally regarded as a country with widespread breastfeeding, the practice continues to be improper as different studies observed $^{43}$. The rate of exclusive breast feeding is also very low in this country ${ }^{45}$. Exclusive breastfeeding in Bangladesh was 63.50 in 2011, according to the World Bank ${ }^{46}$. Some major problems with breast-feeding were identified in a study, regarding exclusive breast feeding, colostrum rejection, initiation of breastfeeding and universal introduction of pre-lacteal feeds ${ }^{47}$. As many as $41 \%$ of infants aged over six months are fed virtually nothing else but breast milk until the age of two years. Bottle feeding is prevalent mainly in the urban areas $^{47}$. One study showed that older women breastfed for a longer duration than their younger counterparts $^{48}$. Another study did not find any significant effect of age on the duration of breastfeeding $^{49}$. Exclusively breastfeeding practice was significantly more among the illiterate mothers in a study, whereas duration of breastfeeding was negatively associated with educational level of mothers in some other studies ${ }^{50,51}$. Poor institutional faccilities reduce the rate of exclusive breast feeding by working mothers ${ }^{52}$. Repeated and organized breast-feeding counselling had a positive impact on exclusive breast-feeding including colostrum feeding, starting complementary feeding and breast feeding during illness like childhood diarrhoea and ARI, described in a study conducted in Bangladesh ${ }^{51}$. Another study showed that, hospital based counseling and campaign cannot ensure exclusive breastfeeding due to delivery of newborns mostly at home ${ }^{53}$.

The child health indicators of Bangladesh are satisfactory in comparison to other South Asian countries. Multiple child health satisfactory programs have been implemented to improve child health and some indicators have shown progress. The infant mortality rate has been brought down to 32 in 1000 live births, however, malnutrition in children under five is still high for many years ${ }^{54}$. With this situation of malnutrition and infant mortality rate in Bangladesh, all possible measures should be taken to support and promote breast-feeding.

\section{Breastfeeding in HIV infection}

In the past few decades, HIV infection became a major challenge for breastfeeding counseling. In African countries, mothers and family members were not allowing breast feeding to newborns and was under much psychological burden. There was critical lack of knowledge amongst health care staff on the transmission of HIV infection from mother to child through breastfeeding ${ }^{17}$. However, the World Health Organization (WHO) issued guidelines to help health workers with infant feeding counseling in the context of HIV. Current guidelines advocate that mothers should exclusively breastfeed their infants 
for the first six months of life, introducing appropriate complementary foods thereafter, and continue breastfeeding for the first 12 months of life ${ }^{55}$. Furthermore, guidelines indicate that prophylactic antiretroviral (ARV) drugs during the breastfeeding period greatly reduce the risk of HIV transmission from mother to infant ${ }^{17}$.

\section{Difficulties regarding breast feeding}

Various forms of difficulties were found for hampering successful breast feeding practices in different studies.

\section{Delayed initiation of breast feeding:}

- Misconception and inadequate knowledge level regarding colostrum delays breast feeding ${ }^{1}$. There is also adequate lack of motivation.

- The other reasons are unusual tiredness to sit up and feed or excessive sleepiness of the baby. But it reflected inadequate motivation of mothers for initiating breastfeeding within one hour of birth.

- One of the major reasons for the delay is sickness of the preterm child and longer staying in tertiary care institute.

- Another reason was that there was delay in shifting from labour room ${ }^{1}$.

\section{Problem with working mother:}

- Mother's employment, unfriendly hospital environment and adverse work related issues act as barriers of breast feeding practice despite knowing benefits of breastfeeding.

- Lack of child care facilities at or near the workplace, rigid time schedules that do not allow for nursing breaks, lack of facilities providing privacy for breast-pumping and absence of facilities for refrigeration of pumped breast milk are important factors that affect breastfeeding prevalence among working mothers ${ }^{18,34}$.

\section{Maternal illness}

- Anemia, stress, and change in lifestyle were mentioned as negative impacts of breastfeeding, on the mother in a Nigerian study, which emphasized requirement of adequate support to overcome stressful changes.
- Breast trauma can be a painful experience for the breastfeeding mother, as well as mental retardation, failure to thrive, and diarrheal disease as disadvantages of breastfeeding.

- Women who had caesarean delivery were more likely not to practice full breastfeeding compared to those who had vaginal delivery, found in a study placed in Jordan ${ }^{17,25}$.

\section{Impression of inadequate milk production}

- Occasionally healthcare providers promote the idea of 'insufficient milk' and encourage women to bottle feed.

- Health care providers often have insufficient skills to offer remedies to deal with breastfeeding problems such as painful or cracked nipples, inflammation or perceived insufficient milk production which often leaves mothers frustrated and leads them to introduce mixed feeding.

- Studies throughout the world have identified that concern about milk supply is the most common reason women give for stopping breastfeeding ${ }^{27,37,51}$.

\section{Availability of formula feed}

- An alarming finding of a high percentage of babies receiving readymade liquid formula while still in hospital, were brought out by a study conducted by Al-Binali.

- Advertisement of breast milk substitutes and ignorance were shown to hinder the optimal practice of breast feeding in different studies. Family pressures and grandparents lack of information on and support for EBF have been also reported as a significant barrier to the continuation of breastfeeding and initiation of formula feeding ${ }^{18,19,34}$.

\section{Cultural and traditional misbelief}

- In African countries specifically in the rural parts, women has a stronger belief in 'bad' milk and are less educated about infant feeding, most likely as a result of poor access to health care.

- The strong role of the extended family in decision making concerning infant feeding, social stigma and inadequate health education provided by health personnel influence on optimal breastfeeding practices and remedies. 
- Fear about the change of breast physiology and sagging of breast resulting from breastfeeding, act as a disadvantage to breastfeeding ${ }^{10,17}$.

Ten Steps to Successful Breastfeeding ${ }^{56}$ : According to guideline of WHO and UNICEF, every facility providing maternity services and care for newborn infants should:

1. Have a written breastfeeding policy that is routinely communicated to all health care staff.

2. Train all health care staff in skills necessary to implement this policy.

3. Inform all pregnant women about the benefits and management of breastfeeding.

4. Help mothers initiate breastfeeding within half an hour of birth.

5. Show mothers how to breastfeed, and how to maintain lactation even if they should be separated from their infants.

6. Give newborn infants no food or drink other than breast milk, unless medically indicated.

7. Practise rooming-in that is allow mothers and infants to remain together - 24 hours a day.

8. Encourage breastfeeding on demand.

9. Give no artificial teats or pacifiers (also called dummies or soothers) to breastfeeding infants.

10. Foster the establishment of breastfeeding support groups and refer mothers to them on discharge from the hospital or clinic.

\section{Recommendations}

Health care professionals can play a vital role in promoting breast-feeding among infants. Studies from different countries have shown that although health professionals had a positive attitude towards breastfeeding but their knowledge was inadequate ${ }^{25}$.

- All Governments should develop and implement a comprehensive policy on infant and young child feeding, in the context of national policies for nutrition, child and reproductive health and poverty reduction ${ }^{1}$.

- Support and counseling by health care workers should be available routinely during ante-natal care, to prepare mothers; at the time of birth to help them initiate breastfeeding; and in the postnatal period to ensure that breastfeeding is fully established ${ }^{1,51}$. Audio visual aids can be provided.
- Medical students, the future physicians will be the first line in dealing with mothers and breastfeeding related problems. The medical curriculum should lay emphasis on counseling regarding maternal and child nutrition ${ }^{4}$.

- Intensive efforts need to be put for the timely initiation of breastfeeding preferably within the labour room itself if there is delay in shifting and the importance of early initiation of breastfeeding needs to be stressed to the mothers in the antenatal period itself ${ }^{1}$.

- Donor expressed breast milk practice should be encouraged for short term in hospitalized patients ${ }^{57}$.

- Mothers need to know how to express their milk so that they can continue to feed their babies and keep up their milk supply if they are separated from their baby" ${ }^{1}$.

- Governments should review progress in national implementation of the International Code of Marketing of Breast Milk Substitutes, and consider new legislation or additional measures as needed to protect families from adverse commercial influences ${ }^{56}$.

- It is speculated that adopting facilitatory measures at hospitals and work place could increase the rate of full breastfeeding. Consideration should be given to provide privacy to nurse mothers in both working and public places ${ }^{25}$.

- Governmental policies regarding longer leave for new mothers and child care centers inside large institutions should be considered ${ }^{30}$.

- Education for women regarding the need for colostrum and education regarding ways of successful breastfeeding can be valuable in decreasing concern about milk supply. The education should be started in antenatal period as well as peer counseling is also recommended ${ }^{1}$.

- Breastfeeding should be linked to public health education for the whole community. Work place breastfeeding policy and lactation rooms are the most acceptable modes for promoting breastfeeding ${ }^{4}$.

- Furthermore, interventions need to be scaled up to empower HIV positive women to practice EBF and to educate family members in conjunction with mothers about the benefits of $\mathrm{EBF}^{29}$. 


\section{Conclusion}

Breastfeeding is of extreme importance for safeguarding health and welfare of the growing infant and this practice must be preserved, protected and promoted by all means. The quality of knowledge and support has a crucial role in the success of breastfeeding promotion. It is well documented that the knowledge and attitude of mothers, family members and healthcare workers towards breastfeeding is far from satisfactory. It is of utmost public health importance that optimal breastfeeding practices, particularly $E B F$, are encouraged to practice in order to promote the growth, survival and health of children. To ensure exclusive breast feeding government of Bangladesh already extended the maternity leave upto 6 months so that all the serving mother can give exclusive breast feeding to their babies. Counseling of mothers on EBF needs to be improved and healthcare workers need to be better trained to provide counseling services.

\section{References}

1. Ekambaram M, Bhat VB, Ahamed MAP. Knowledge, attitiude and practice of breastfeeding among postnatal mothers. Curr Pediatr Res 2010; 14(2):119-24.

2. World Health Organization. Infant and young child feeding Model Chapter for textbooks for medical stu-dents and allied health professionals. World Health Organization. 2009. Available from whqlibdoc.who.int/publications/2009/ 9789241597494_eng.pdf.

3. Bahl R, Frost C, Kirkwood BR, Karen E, Martines J, Bhandari $\mathrm{N}$ et al. Infant feeding patterns and risks of death and hospitalization in the first half of infancy: multicentre cohort study. World Health Organization 2005; 83:418-26.

4. Anjum Q, Ashfaq T, Siddiqui $H$. Knowledge regarding Breastfeeding Practices among Medical Students of Ziauddin. JPMA 2007; 57(10):480-7.

5. Gupta A, Arora V, Bhatt B. The State of World's Breastfeeding: India Report card 2006. International Baby Food Action Network (IBFAN), Asia Pacific. India. 2006. Accessed from http://www.internationalbreastfeedingjournal. com/content/3/1/5

6. Dadhich JP, Gupta A. Assessment of Status of Infant and Young Child Feeding (IYCF) practice, policy and program-Achievements and Gaps. Breast feeding promotion network of India. 2005. Available from www.iosrjournals. org/iosr-jdms/papers/Vol14.1/T014517780.pdf.

7. Community-Based Strategies for Breastfeeding Promotion and Support in Developing Countries. Geneva, Switzerland: World Health Organization; [updated 2003]. Available from http://www.Linkagesproject.org/media/publications /Technical20Reports/CommunityBFStrategies.pdf.

8. Thulier D, Mercer J. Variables associated with breastfeeding duration. J Obstet Gynaecol Neonatal Nurs 2009; 38(3):259-68.
9. Nabulsi $\mathrm{N}$ : Why are breastfeeding rates low in Lebanon? a qualitative study. BMC Pediatr 2011; 11:75-80.

10. Knowledge, attitudes and practices regarding exclusive breastfeeding in Southern Africa - Part 1. Written by Rita Magawa 2012. Available from http://www. polity. org.za/article/Knowledge-attitudes-and-practices-regardingexclusive-breastfeeding-in-Southern-Africa-Part 1-2012-12-05.

11. Library of evidence for nutrition actions (eLENA): Exclusive breastfeeding', World Health Organization, December 2011. Available from http://www.who.int.

12. Maternal, newborn, child and adolescent health: Breastfeeding', World Health Organization. Available from http://www.who.int

13. WHO. The optimal duration of exclusive breastfeeding: report of an expert consultation. Geneva: World Health Organization; 2001. Available from www.who.int/nutrition/ publications/Complementary_Feeding.pdf.

14. 'Exclusive breastfeeding for six months best for babies everywhere', World Health Organization, January 2011. Available from http://www.who.int

15. Breastfeeding on the Worldwide Agenda: Findings from a landscape analysis on political commitment for programmes to protect, promote and support breastfeeding. Available from www.unicef.org.

16. Al-Binali AM. Knowledge, attitude and practice of Breast-Feeding among female health care workers in tertiary care hospitals. The Medical Journal of Cairo University 2012; 80(1):159-64

17. Utoo BT, Ochejele S, Obulu MA, Utoo PM. Breastfeeding Knowledge and Attitudes amongst Health Workers in a Health Care Facility in South-South Nigeria: the Need for Middle Level Health Manpower Development. Clinics in mother and child health 2012; 9:1-5.

18. Al-Binali AM. Breastfeeding knowledge, attitude and practice among school teachers in Abha female educational district, southwestern Saudi Arabia. International Breastfeeding Journal 2012; 7(9):10-2.

19. Uchendu U, Ikefuna A, Emodi I. Factors associated with exclusive breastfeeding among mothers seen at the University of Nigeria Teaching Hospital. SAJCH 2009; 3:14-9.

20. Brady JP. "Marketing breast milk substitutes: problems and perils throughout the world". Archives of Disease in Childhood 2012; 97(6):529-32

21. Infant formula or breast milk? Available from www.promotebmscode.com

22. '10 facts on breastfeeding', World Health Organization, July 2012. Available from http://www.who.int.

23. Cai X, Warldlaw T, Brown DW. Global trends in exclusive breastfeeding. Int Breastfeed J 2012; 7:7-12.

24. Khan ME. Breast feeding and Weaning Practices in India. Asia Pac Popul J 1990; 5(1):71-88.

25. Khassawneh M, Khader Y, Amarin Z, Alkafajei A. Knowledge, attitude and practice of breastfeeding in the north of Jordan: a cross-sectional study. International Breastfeeding Journal 2006; 1:1-17. 
26. Chatman LM, Salihu HM, Roofe ME, Wheatle P, Henry $D$, Jolly PE. Influence of knowledge and attitudes on exclusive breastfeeding practice amorng rural Jamaican mothers. Birth 2004; 31(4):265-71.

27. Arts M. Knowledge, beliefs, and practices regarding exclusive breastfeeding of infants younger than six months in Mozambique: A qualitative study. Journal of Human Lactation 2011; 27(1):25-32.

28. Fjeld E. "No sister, the breast alone is not enough for my baby": A qualitative assessment of potentials and barriers in the promotion of exclusive breastfeeding in southern Zambia. International Breastfeeding Journal 2008; 3:26-38.

29. SN Okolo AZ, YB Adewunmi B, MC Okonji C. Current breastfeeding knowledge, attitude, and practices of mothers in five rural communities in the Savannah region of Nigeria. J Trop Pediatr 1999; 45(6):323-26.

30. Jalil AM, Ibrahim M, Yusof NFM, et al. Knowledge, attitude and practices of breast feeding among working mothers at University Sultan Zainal Abidin (UNISZA), Kuala Terengganu, Malaysia. Available from www.kenes.com/ apccn/scientific/Documents/Poster/481.pdf.

31. Agarwal S, Srivastava K, Sethi V. Maternal and New-born Care Practices Among the Urban Poor in Indore, India: Gaps, Reasons and Possible Program Options. Urban Health Resource Center, New Delhi. 2007. Available from www.uhrc.in.

32. Petit Al. Perception and knowledge on exclusive breastfeeding among women attending antenatal and postnatal clinics. A study from Mbarara Hospital-Uganda, August 2008. Dar Es Salaam Medical Student's Journal 2010; 16(1):27-30

33. Meedya S, Fahy K, Kable A. Factors that positively influence breastfeeding duration to 6 months: A literature review. Women Birth 2010; 23(4):135-45.

34. Sadoh AE, Sadoh WE, Oniyelu P. Breast feeding practice among medical women in Nigeria. Niger Med $\mathrm{J}$ $2011 ; 52: 7-12$

35. Bezner-Kerr RM. "We grandmothers know plenty": Breastfeeding, complimentary feeding and the multifaceted role of grandmothers in Malawi. Social Science \& Medicine 2007; 66:1095-1105.

36. Narayan S, Natarajan N, Bawa KS. Maternal and Neo-natal Factors Adversely Affecting Breastfeeding in the Perinatal Period. Med J Armed Forces Ind 2005; 61 216-19.

37. Scott JA, Aitkin I, Binns CW, Aroni RA. Factors associated with the duration of breastfeeding amongst women in Perth, Australia. Acta Paediatr 1998; 88(4):416-21.

38. Li R, Hsia J, Fridinger F, Hussain A, Benton-Davis S, Grummer-Strawn L. Public beliefs about breastfeeding policies in various settings. J Am Diet Assoc 2004; 104: 1162-8.

39. Subbiah N. A Study to assess the Knowledge, Attitude, Practice and Problems of Postnatal Mothers regarding Breastfeeding. Nursing J Ind 2003; 94(8):177-9.
40. Al-Jassir MS, El-Bashir BM, Moizuddin SK, Abu-Nayan AA. Infant feeding in Saudi Arabia: mothers' attitudes and practices. East Mediterr Health J 2006; 12(1-2):6-13.

41. Talukder MQ-K, Hassan MQ. Maternal nutrition and breastfeeding. Bangladesh J Child Health 1992; 16:95-8.

42. Jones G. How many child deaths can we prevent this year? Lancet 2003; 362:65-71.

43. Baqui $\mathrm{AH}$, Paljor $\mathrm{N}$, Nahar $\mathrm{Q}$, Silimperi DR. Infant and child feeding practice in Dhaka urban slums. Dhaka: International Centre for Diarrhoeal Disease Research, Bangladesh 1993; 34(6):9-14.

44. Kawser CA, Hassan MQ, Kabir ARML. Scientific evidence on the benefits of breast feeding. J Bangladesh Coll Phys Surg 1995; 14:29-37.

45. Islam MD, Islam MS, Shaha SK. Changing trends in Infectious Diseases in Children, Dhaka Shishu hospital Experience. DS (Child) 2003; 10(1):5-10.

46.http://www.tradingeconomics.com/bangladesh/exclusiv e-breastfeeding-percent-of-children-under-6-months-webdata.html

47. Akhter $\mathrm{HH}$, Akhter M, Azad KMR. A national baseline survey to assess the breastfeeding practices in Bangladesh, Dhaka: Bangladesh Institute of Research for Promotion of Essential \& Reproductive Health and Technologies 1998; 1:151-6.

48. Ahmad S, Alam MS. Determinants of breastfeeding in an urban area of Bangladesh. J Fam Welfare 1996; 42:1-6.

49. Ahamed MM. Breast-feeding patterns in Bangladesh. J Fam Welfare 1988; 34:36-44

50. Islam MN, Islam MM. Breast-feeding pattern in Bangladesh. In: Proceedings of the Fourth National Conference and Seminar on "Prevention of Maternal and Child Malnutrition," Dhaka, 19 May 1992. Dhaka: Bangladesh Population Association1992; 209-18.

51. Haque MF, Hussain M, Sarkar AK, Hoque MM, Ara FA, Sultana S. Breast-feeding Counselling and Its Effect on the Prevalence of Exclusive Breast-feeding. J Health Popul Nutr 2002; 20(4):312-6.

52. Haider R, Begum S, Journal of Human Lactation 1995; $11(4): 273-7$

53. Haider R, Ashworth A, Kabir I, Hutty SRA. The Lancet 2000; 356(9242):1643-7.

54. Geoba.se: Gazetteer - Egypt - 2040 - Statistics and Rankings.www.geoba.se/country.php?

55. World Health Organization, Guidelines on HIV and infant feeding 2010, WHO, Geneva, 2010, www.who.int/maternal child_adolescent/documents/9789241599535/en/index.html.

56. Global strategy for infant and young child feeding', World Health Organization, 2003. Available from http://www.who.int.

57. Donor Breast Milk Banks: The Operation of Donor Milk Bank Services. NICE Clinical Guidelines, No. 93. Available from www.nice.org.uk/Guidelines Manual 